\title{
Recombination suppressors and the evolution of new species
}

\author{
ADAM J. TRICKETT* \& ROGER K. BUTLIN \\ Department of Genetics, University of Leeds, Leeds LS2 9JT, U.K.
}

\begin{abstract}
Chromosomal rearrangements are often the only apparent difference between closely related species, although it is not clear whether they are a cause or a by-product of speciation. We suggest that changes in the pattern of recombination may provide a link between chromosomal rearrangements and speciation. In models of speciation by sexŭal selection and by reinforcement, recombination is a major barrier to the formation of new species, primarily because it opposes the establishment of linkage disequilibrium. Here we show that in both the Felsenstein (1981) and Kirkpatrick (1982) models, a recombination suppressor is able to enhance the processes leading to speciation and increase its own frequency in the population.
\end{abstract}

Keywords: chromosomal rearrangements, recombination suppression, reinforcement, sexual selection, speciation.

\section{Introduction}

It has long been observed that chromosomal rearrangements are associated with the formation of new species. Closely related species frequently differ chromosomally despite high genetic similarity (White, 1978; King, 1993). Some species apparently only differ for a few genomic rearrangements (Lewis, 1973). It has been suggested that the chromosomal rearrangements are underdominant and thus play a direct part in the evolution of reproductive isolation, although it is difficult to explain their local fixation and spread in this case (Lande, 1979; Barton \& Hewitt, 1980). Underdominance produced by some type of single rearrangements is, in fact, often very weak or absent, e.g. inversions in Drosophila (Sturtevant \& Beadle, 1939; Coyne, 1992), fusions in Sorex (Searle, 1986) or Podisma (Hewitt \& Barton, 1980) and so additional reasons for the association between chromosomal change and speciation need to be sought.

A common feature of chromosomal rearrangements is modification of the pattern of recombination: inversions cause local suppression of recombination whereas fusions and translocations bring into linkage previous unlinked loci. This suggests a possible link with speciation as Felsenstein (1981) has identified recombination as a primary process opposing progress towards speciation. This is a problem common to all

${ }^{*}$ Correspondence. models of speciation by reinforcement (Butlin, 1987) which require the build-up of linkage disequilibrium between underdominant loci and assortative mating loci.

Similarly, models of speciation by sexual selection (O'Donald, 1962; Lande, 1981; Kirkpatrick, 1982; Seger, 1985; Maynard Smith, 1991) rely on linkage disequilibrium, in this case between male trait loci and female preference loci. The greater the linkage disequilibrium maintained by assortative mating, the greater the probability of rapid divergence in secondary sexual traits and preferences by the Fisher process. Recombination influences both the build-up and decay of disequilibrium in these models and thus influences evolutionary trajectories. Divergence in traits and preferences can, of course, lead to prezygotic isolation between populations. The importance of linkage disequilibrium is not restricted to the Fisher process but this is the mechanism of sexual selection that has been most widely discussed in the context of speciation (e.g. West-Eberhard, 1983).

We have therefore extended the Kirkpatrick and Felsenstein models by including a suppressor of recombination. This suppressor will be described as an inversion for the sake of brevity but other chromosomal rearrangements may have similar effects, as do many nonchromosomal suppressors of recombination. The Kirkpatrick model is used as an example of a sexual selection model because its simplicity makes the effects of recombination suppression easy to visualize. The 
likely outcome in more complex, especially polygenic, models will be discussed below.

For each model we consider: (i) whether the inversion will spread, and (ii) whether its spread alters the likelihood of progress towards speciation. Both computer models were developed using Borland Turbo Pascal 5.5. The Kirkpatrick model was written from scratch but the Felsenstein model was a modified version of Felsenstein's (1981) program, obtained from him. Source code and listings are available upon request. Each model was tested against the original author's data to demonstrate concordance with his numerical results when no inversion was present.

\section{Felsenstein model}

The model developed by Felsenstein is a very simple population simulation. The population is infinite but is divided into two subpopulations, with a variable level of migration, $m$. The organism is haploid, with only three loci of interest. The $A$ locus influences assortative mating: $A$ females prefer $A$ males and $a$ females prefer $a$ males. A parameter, $d$, specifies the strength of assortative mating, ranging from 1.0 for complete assortative mating to 0.0 for random mating. The other two loci, $B$ and $C$ are under selection, the $B C$ genotype being at a selective advantage in one subpopulation and $b c$ being at an advantage in the other. The selection, $s$, is symmetrical and multiplicative, and higher values of $s$ represent stronger selection against individuals in inappropriate environments (see Felsenstein (1981) for further details).

Felsenstein found that progress towards speciation, measured by the establishment of disequilibrium between the $A$ locus and the $B$ and $C$ loci, is favoured by strong selection, strong assortative mating, low migration and low recombination between the assortative mating locus and the first selected locus (between $A$ and $B$, given the notional genetic map $A B C$ ). For any set of conditions, there is a threshold value of assortative mating above which the system is able to maintain strong positive disequilibrium. The threshold is lower for high values of selection and low values of recombination (Figs 1 and 2). Felsenstein concluded that recombination was a major factor opposing progress towards speciation.

Adding an inversion, that suppresses recombination between the $A$ and $B$ loci, to a proportion of the $A B C$ individuals in the starting population bears out the observation from the original model: reducing the amount of recombination between the $A$ and $B$ loci increases the range of conditions in which disequilibrium is established (Figs 1-3). The strength of assortative mating required to obtain stable disequilib-

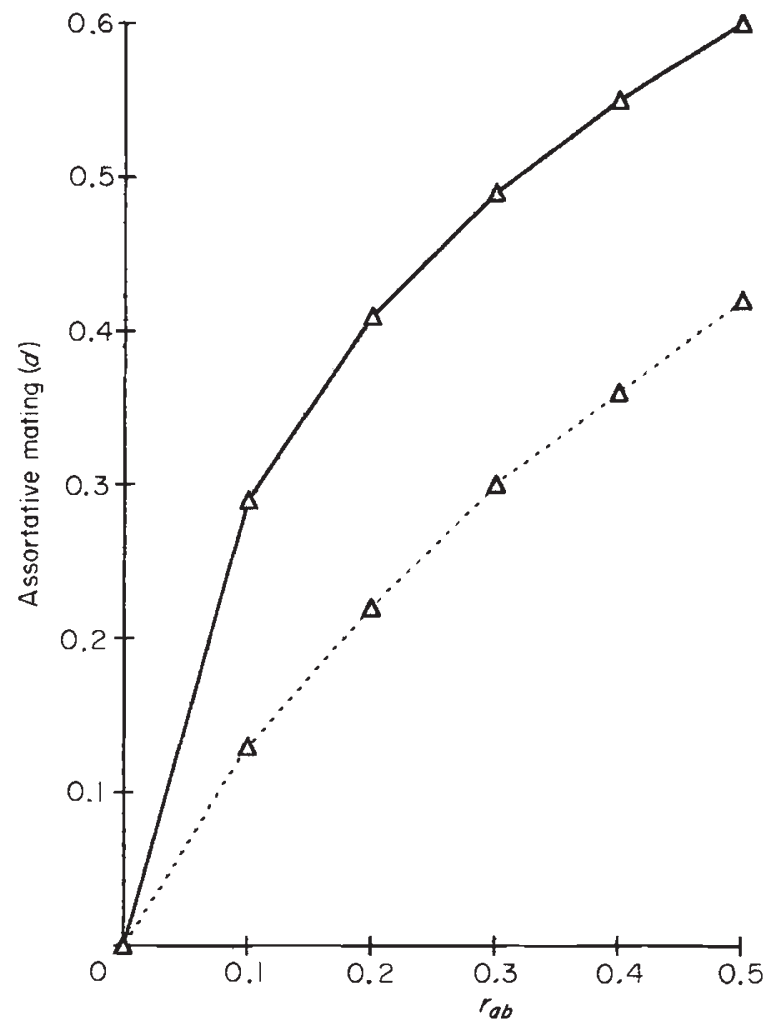

Fig. 1 Effect of recombination between the $A$ and $B$ loci on the minimum amount of assortative mating required for a stable nonzero level of $A B C$ linkage disequilibrium in the Felsenstein model. The solid line is the basic Felsenstein model, the dashed line is the case where the inversion is present over the $A$ and $B$ loci in 25 per cent of the $A B C$ genotype at the start of the run. Migration $(m)=0.1$, Selection $(s)=1.0$ and recombination $\left(r_{A B}\right.$ and $\left.r_{B C}\right)$ between each pair of loci in the absence of the inversion $=0.5$.

rium drops substantially although there is little effect on the final level of disequilibrium. The effect of the inversion is most pronounced where the selection coefficients are intermediate, and most realistic, and when recombination is initially high.

The inversion was observed to spread such that all $A$ individuals carried the inversion. Once this situation has been reached, no recombination between $a b$ and $A B$ genotypes can occur and so the level of disequilibrium is maintained indefinitely.

Felsenstein's results were independent of starting conditions. The arbitrary starting conditions chosen for our simulations were: all allele frequencies $0.5, A$ and $B$ together 75 per cent of the time and $A$ and $C$ together 75 per cent of the time; thus $A B C$ and $a b c$ are represented by 28.125 per cent of the initial population. However, the initial frequency of the inversion as a proportion of $A B C$ individuals did influence the outcome. Even very small initial inversion frequencies 


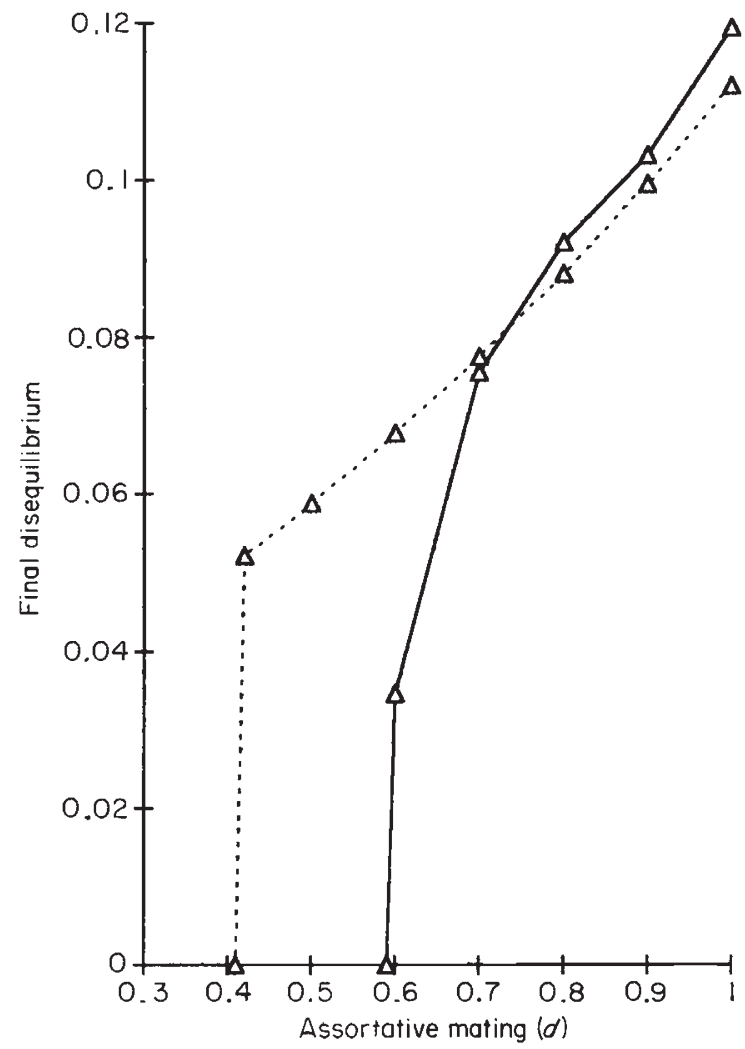

Fig. 2 Effect of assortative mating on the final stable level of linkage disequilibrium. Stability assumed after 15,000 generations of no change in the value of $A B C$ gametic linkage disequilibrium. Parameters and conventions as in Fig. 1. Disequilibrium is measured over the whole $A B C$ region, by following the pairwise linkage disequilibrium between loci $A$ and $C$, as in Felsenstein (1981).

were observed to reduce the critical value of assortment for a given value of selection (Fig. 4). If the inversion were to arise while the $A B C$ genotype was itself rare, then in a finite population it may represent a substantial proportion of that genotype and thus produce a large increase in the probability of speciation.

When the inversion suppressed recombination between the $B$ and $C$ loci, progress towards speciation was impaired as expected. Felsenstein found this to be the result of decreasing recombination in his original model because it is the production of unfavourable combinations of $B$ and $C$ alleles that generate selection for assortative mating. The inversion itself was able to sweep throughout both subpopulations, eliminating all noninverted $B C$ genotypes. This eliminates the production of $b C$ and $B c$ gametes and thus the selection for linkage disequilibrium with the $A$ locus, i.e. for reinforcement.

This is an effect of the haploid model used and was absent in Felsenstein's diploid model where selection

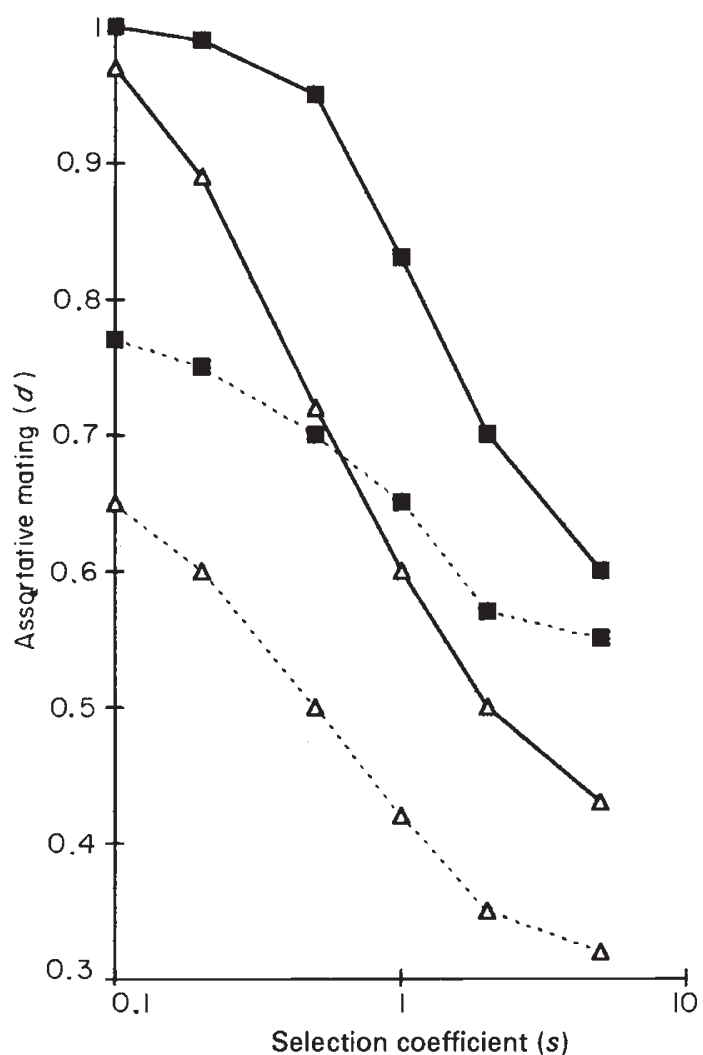

Fig. 3 Minimum amount of assortative mating $(d)$ required to establish stable nonzero linkage disequilibrium between the locus $A$ and the loci $B$ and $C$, plotted for various selection coefficients in the Felsenstein model. The squares are for $m=0.5$, the triangles are for $m=0.1$. The symbols interpolated on the solid line are for no inversion and the symbols interpolated on the dashed line are where the inversion was present at 0.25 of the $A B C$ genotypes at the beginning of the run. $r_{A B}=r_{B C}=0.5$, run length up to 250,000 generations.

against 'hybrids' was not dependent on epistatic interactions between loci. We would not expect the suppression of recombination among selected loci in a diploid model to impair speciation. On the other hand, Felsenstein found that recombination between the mating locus and the selected loci had the same effect in his diploid model as in the haploid version. Therefore, we expect that the observed effect of a recombination suppressor on progress toward speciation is not limited to the haploid case.

Other possible positions for the inversion have predictable effects. $\underline{A B C}$ or $a b c$ and $\underline{A b} c$ or $\underline{a B C}$ are all equivalent (where underscore represents the inverted section). Starting combinations such as $\underline{A B} c$, in which the inversion is initially linked to a disadvantageous combination of $B$ and $c$ alleles, quickly generate by recombination a favoured genotype, $A B C$, and are analogous to starting with the inversion in the favoured genotype, only at a lower initial frequency. All inver- 


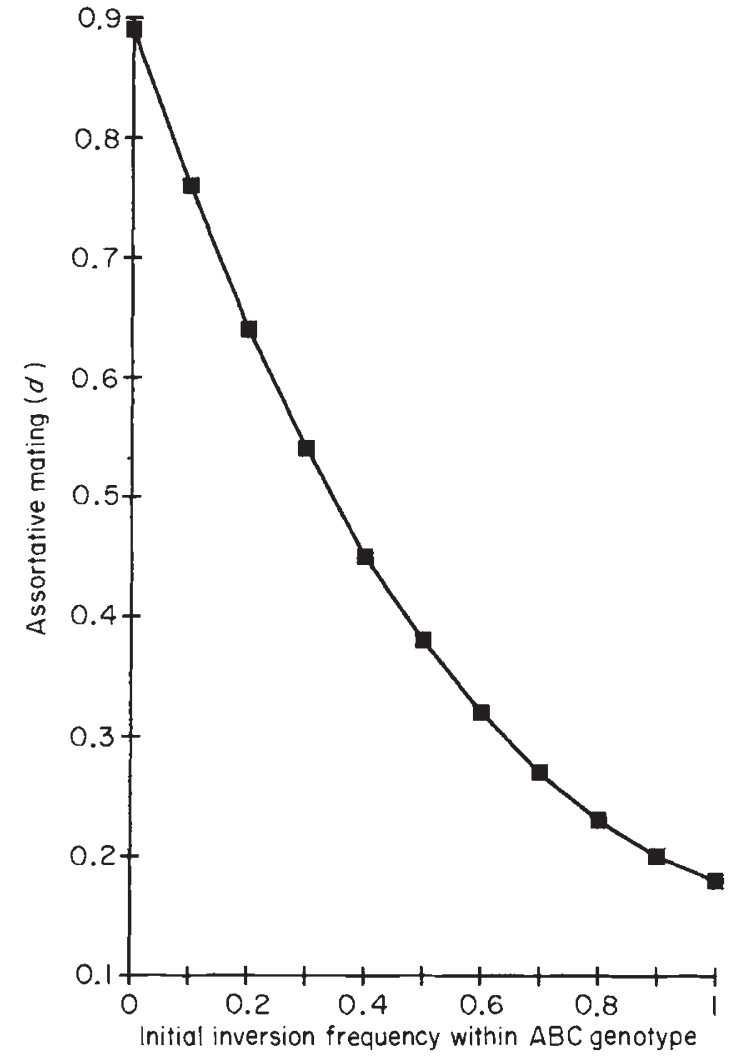

Fig. 4 Minimum amount of assortative mating $(d)$ required to obtain stable association between the locus $A$ and the loci $B$ and $C$, plotted for various initial inversion frequencies as a proportion of the $A B C$ genotype, in the Felsenstein model. Given that $s=0.2, r_{A B}=r_{B C}=0.5$ and $m=0.1$.

sions over the $B C$ region hinder progress towards speciation, although they themselves are spread throughout the population if they include a favourable combination of alleles.

Diehl \& Bush (1989) modified the Felsenstein model by the inclusion of habitat-associated mating and found that this eased the conditions for establishment of disequilibrium. Suppression of recombination may combine with this effect to increase the probability of speciation still further.

\section{Kirkpatrīck model}

In the Felsenstein model prezygotic isolation can be generated by reinforcement. However, prezygotic isolation may also result from divergence of mating traits and preferences, due to sexual selection in allopatric populations. We have modified the Kirkpatrick (1982) model of sexual selection rather than the Lande (1981) model because of its relative simplicity but the congruent conclusions from the two original models suggest that suppression of recombination would have qualitatively similar effects (see below). In the Kirkpatrick model only two loci are considered, $P$ and $T . P_{I}$ females mate at random, while $P_{2}$ females mate assortatively, $\alpha_{2}$ times more frequently than at random. $T_{1}$ males are normal, while $T_{2}$ males possess a secondary sexual trait that incurs a selective disadvantage of $S$. The population is infinite, the organisms are haploid and no other effects are considered.

The model produces a simple deterministic result, with populations approaching a line of neutral equilibria, occasionally leaving both loci polymorphic. The likelihood of fixation of the trait allele is: (i) inversely proportional to the selection against trait-bearing males, (ii) directly proportional to the strength of female preference, (iii) dependent on initial preference and trait allele frequency, and (iv) influenced by the recombination fraction. The effect of recombination is limited to the initial evolutionary trajectory and is small, except when there is no recombination. This effect operates through the level of positive linkage disequilibrium generated by assortative mating. If there is a cost associated with preference in females, then the outcome is always elimination of both $P_{2}$ and $T_{2}$ alleles (but see Pomiankowski et al., 1991).

The line of neutral equilibria is an attractor, to which the allele frequencies move. Above the line, preference and trait both increase, below the line they both decrease and on the line the system is static. The position of the line is independent of the recombination frequency $(r)$ between $P$ and $T$ but recombination influences disequilibrium between $P$ and $T$ and thus the slope of evolutionary trajectories for populations away from equilibrium. In agreement with these observations, an inversion, suppressing recombination between the $P_{2}$ and $T_{2}$ alleles has no detectable effect on the position, gradient or stability of the line of neutral equilibria. It does, however, affect the linkage disequilibrium between the alleles and hence their behaviour away from equilibrium. It increases the correlation between the trait and the preference alleles such that they reach the line of neutral equilibria at a much higher position (Figs 5 and 6). In many cases it is possible for the trait or both trait and preference alleles to be fixed in the population. The inversion is, in effect, increasing the genetic regression of preference on trait $(B / G$ ratio) which specifies the evolutionary trajectory followed by a population in both the Kirkpatrick and Lande (1981) models.

Figures 5 and 6 clearly demonstrate the increased correlation between $P_{2}$ and $T_{2}$ caused by the inversion generating and maintaining a higher level of linkage disequilibrium. A similar effect can be produced initially by reducing the overall amount of recombination but even tiny amounts of recombination do 


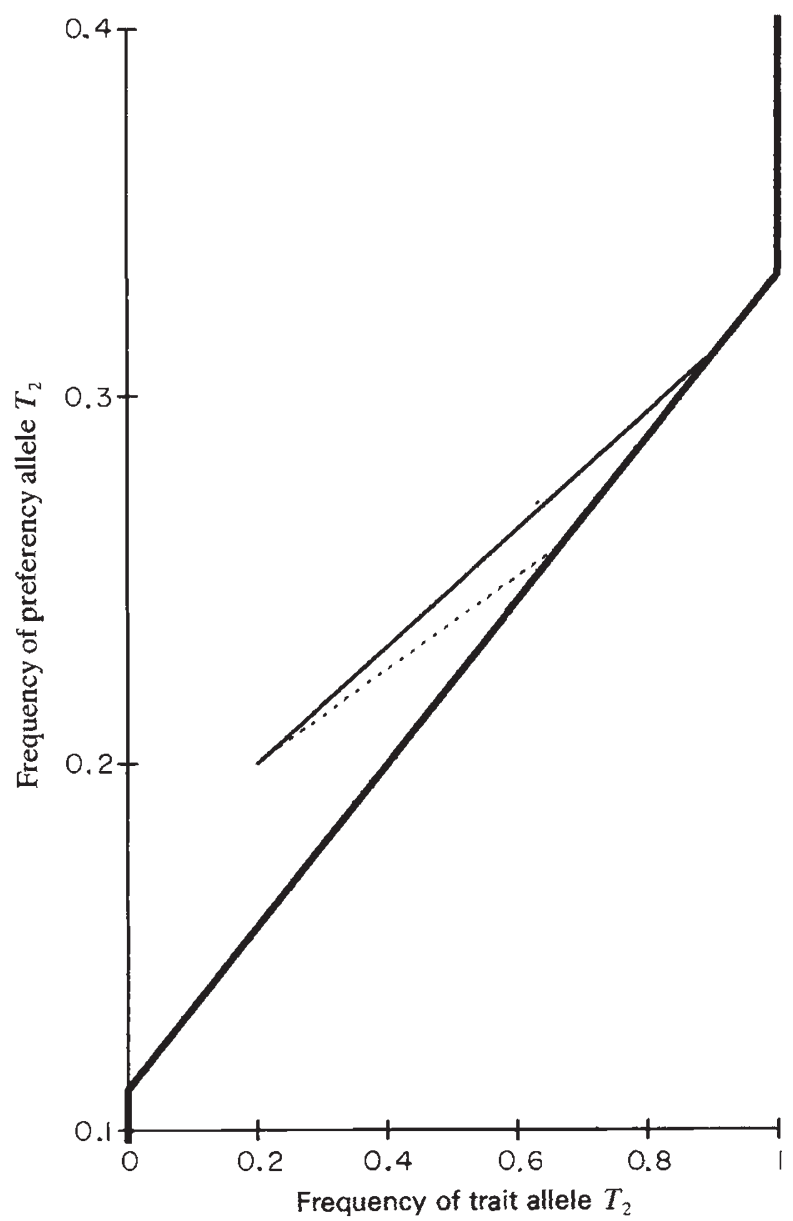

Fig. 5 Effect of an inversion on the trajectories of the allele frequencies in the Kirkpatrick model. The inversion does not alter the location of the line of neutral equilibria (heavy curve). The gradient of the line of neutral equilibria is given by the expression: $S\left(1+\alpha_{2} S-\alpha_{2}\right) /(S-1)\left(\alpha_{2}-1\right)$ and intercepts the $y$ axis at the point: $-S /\left(-\alpha_{2}+1+\alpha_{2} S-S\right)$. The inversion raises the gradient of the trajectory, making fixation of the trait allele or both trait and preference alleles more likely, in any given situation. In this example the starting conditions were: $T_{2} P_{2}$ frequency $0.1 ; T_{2} P_{1}$ frequency $0.1 ; T_{1} P_{2}$ frequency $0.1 ; T_{1} P_{1}$ frequency $0.7 ; S=0.25$; $\alpha_{2}=4.0$ and $r_{P T}=0.5$. The lower dashed line is for the case without an inversion, and the higher solid line is for the case when $P_{2} T_{2}$ had an inversion representing 10 per cent of its frequency.

quickly break down the association and the alleles return to their uncorrelated state.

With higher levels of linkage disequilibrium generated, any perturbation from the line of neutral equilibria, by processes such as drift or environmental change, would result in the system moving further away from the line than would be possible otherwise. Thus progress towards fixation of $P_{2}$ and $T_{2}$ becomes more probable when a population is displaced above the line but elimination of these alleles becomes more probable following displacements below the line (Fig. 7).
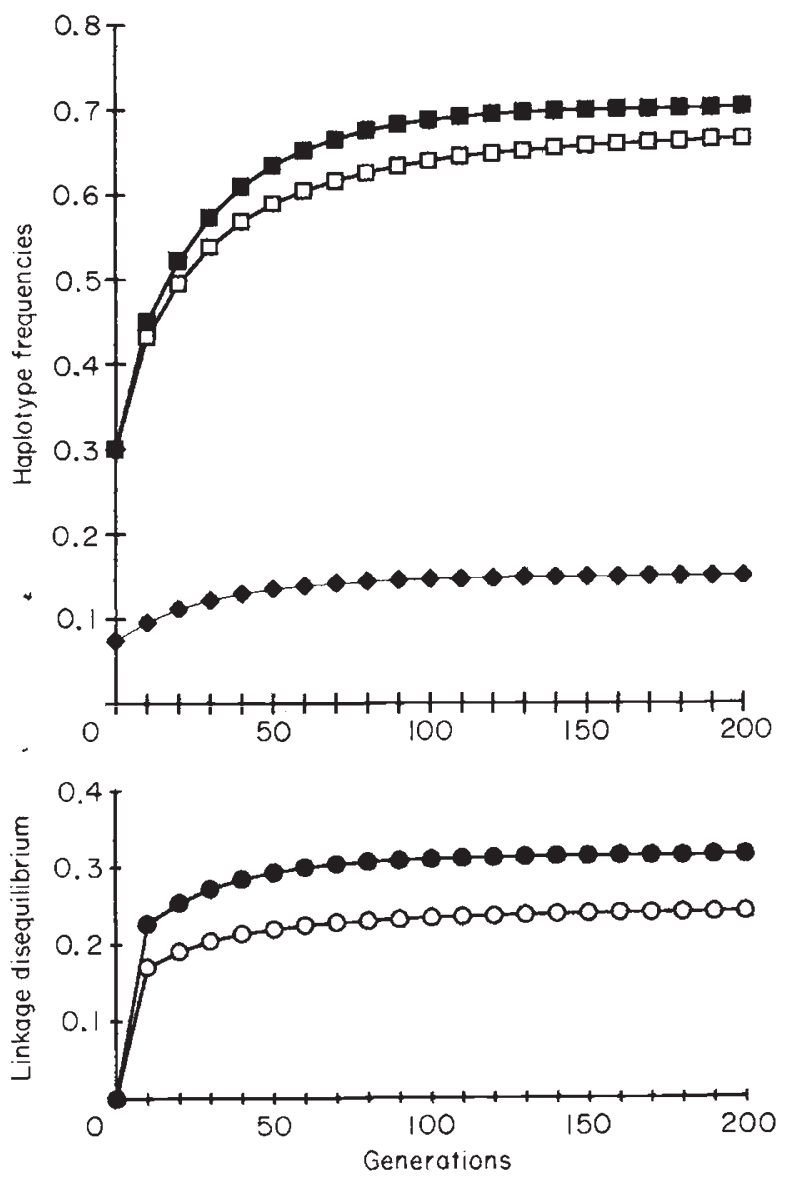

Fig. 6 Upper graph is a plot of the haplotype frequencies versus generation time in a typical run, the lower graph shows the linkage disequilibrium for the same run. Initial values: $P_{2} T_{2}=0.3 ; P_{1} T_{2}=0.2 ; P_{2} T_{l}=0.3 ; P_{l} T_{l}=0.2 ; S=0.5$; $\alpha_{2}=4 ; r_{P T}=0.5$; inversion present as 25 per cent of $P_{2} T_{2}$ in the inverted case. These starting conditions were chosen to ensure that the system was initiated above the line of neutral equilibria. In both plots the solid symbols refer to the inverted case, the clear symbols refer to the noninverted case. In the upper graph the box is the frequency of $P_{2} T_{2}$ and the diamond is the frequency of the inversion. Disequilibrium was calculated as $d / d_{\max }$ and thus has a range of -1 to +1 .

The frequency of the inversion only increases in the overall population as a proportion of the genotype in which it is present. Thus if initially present at 20 per cent of the $P_{2} T_{2}$ genotype, it will still exist at 20 per cent of the $P_{2} T_{2}$ genotype but the frequency of $P_{2} T_{2}$ will have risen and thus the absolute frequency of the inversion, within the population as a whole, will have risen (see Fig. 6). Kirkpatrick made the important point that the increase in frequency of $P_{2}$ as the $T_{2}$ allele spread to fixation may favour the spread of subsequent $T$ alleles that further increased the preferred trait. Similarly, the increase in frequency of the inversion will increase the likelihood of tight linkage between the preference allele and new trait alleles appearing within the inversion, 

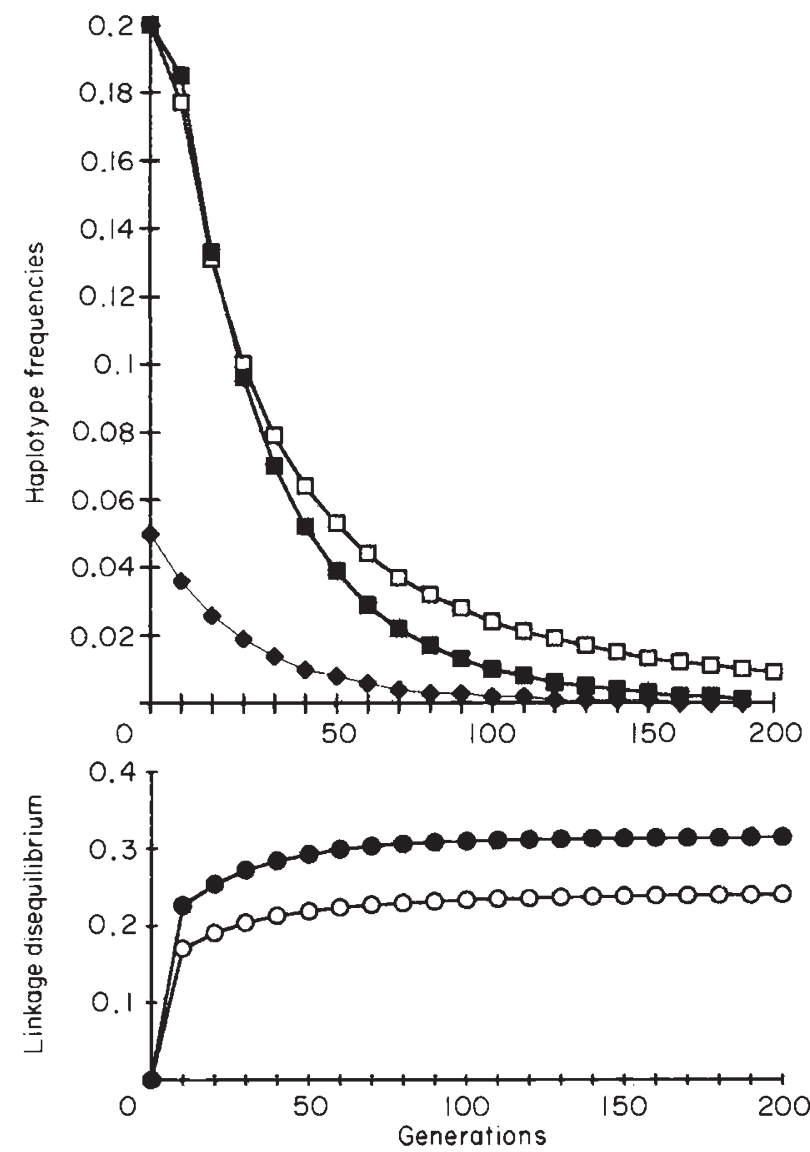

Fig. 7 Typical run as in Fig. 6 except that here the system was initiated below the line of neutral equilibria, all other starting conditions being identical. The symbols are the same as Fig. 6. Initial frequencies: $P_{2} T_{2}=0.2, P_{l} T_{2}=0.3, P_{2} T_{I}=0.2$ and $P_{l} T_{l}=0.3$.

further enhancing their chances of fixation. New trait alleles appearing at loci outside the inversion will gain the advantage of pre-existing female preference.

The inversion was introduced into each of the four possible genotypes. The results for $P_{2} T_{2}$ have been described. If introduced into the $P_{1} T_{1}$ genotype, the effect of the inversion is qualitatively identical as disequilibrium is similarly enhanced. The $P_{1} T_{2}$ and $P_{2} \hat{T}_{1}$ combinations give lower levels of linkage disequilibrium but otherwise no major change to the behaviour of the model. In neither case was the frequency of the inversion seen to rise, although only limited runs of these types were carried out.

In a polygenic model such as Lande's (1981), the effect of an individual suppressor of recombination would be less as it would encompass only a fraction of the loci involved in preference and trait determination. On the other hand, a greater incidence of inversions or other rearrangements including at least some relevant loci would be expected. As in the Kirkpatrick model, an inversion would only enhance population divergence if it happened to hold together appropriate preference and trait alleles. These combinations are expected to be in higher frequencies than otherwise expected because of assortative mating but clearly the likelihood of a significant effect will depend on the distribution of loci.

\section{Discussion}

Two types of model for the behaviour of inversions, or recombination suppressors in general, in populations have been explored in the past. Some consider the spread of a rearrangement by drift in small populations despite underdominance. This process may be aided by meiotic drive or by selection favouring the mutant homozygote but, nevertheless, conditions for both the local fixation (Lande, 1979) and spread (Barton \& Rouhani, 1991) of the rearrangement are very stringent. Others (Fraser \& Burnell, 1967; Charlesworth \& Charlesworth, 1973) have attempted to model the spread of inversions as a consequence of their effects on recombination. These models show that the deterministic spread of an inversion from low frequency is possible, although it remains unlikely.

Fraser \& Burnell (1967) considered the effect of introducing an inversion into a multilocus system without epistasis but did not find conditions for the spread of the rearrangement when rare. Charlesworth \& Charlesworth (1973) found that epistatic fitness interactions are a necessary condition for the spread of a rearrangement in a multilocus system. A rearrangement will increase in frequency from a low starting value only if the population is at or near an equilibrium with linkage disequilibrium. They found that there are several types of fitness interaction that can generate stable equilibria with little or no linkage disequilibrium for certain ranges of the recombination parameters and, in such cases, there is virtually no selection in favour of a new inversion, unless the initial inversion frequency is above a critical threshold value.

The models we describe involve the introduction of inversions into systems that maintain disequilibrium because of assortative mating and so the spread of the inversion that we observe is consistent with the conclusions of Charlesworth \& Charlesworth (1973). The interesting additional feature of these models, however, is that the spread of the inversion potentially increases the extent of reproductive isolation between populations. It may contribute to speciation and thus help to explain the observed association between chromosomal rearrangements and speciation events.

Both the Kirkpatrick and Felsenstein models are designed to be simple, ignoring the complexities of real systems, so that they can be used to explore the key elements of natural systems. Both models have shown the importance of recombination as a process that controls linkage disequilibrium. Thus the suppression of recombination may be a critical element in specia- 
tion models involving either sexual selection or reinforcement.

Our results show that the suppressor has a greater effect, and itself increases to a higher frequency, in the reinforcement model than in the sexual selection model. In both cases the effects are likely to be reduced by polygenic determination of the traits involved when the distribution of loci will be an important factor. The availability of recombination suppressors will also be a critical factor but may not be limiting since observed rates of chromosomal mutation are high $(>1$ in 500 gametes; King, 1993).

A test of the predictions of these recombination suppressor models is simple in principle, though difficult in practice. The theory predicts that 'speciation' genes should be located in rearranged zones of the genome or more tightly linked than their physical distance might suggest. However, at present very few loci involved in speciation have been identified, let alone mapped (Coyne, 1992; Ritchie, 1992). There is one example of sexual selection in which preference and trait genes are apparently both associated with a complex inversion: in the seaweed fly, Coelopa frigida (Gilburn et al., 1992) but this inversion is polymorphic over a very wide range and does not appear to be involved in divergence among populations.

Genes for both signal traits and preferences that distinguish two closely related sulphur butterflies, Colias eurytheme and C. philodice, have been localized to the $\mathrm{X}$ chromosome. An ultraviolet wing marker, two distinct pheromone systems, and their associated female preferences, are all controlled by major genes located on the X chromosome (Grula \& Taylor, 1980). This pattern is unlikely to have arisen by chance in an organism with a large number of chromosomes: it may be the result of selection for linkage as predicted by our models and as suggested by Grula and Taylor.

\section{Acknowledgements}

We wish to thank J. Felsenstein for the use of his source code and helpful explanations, and two anonymous referees for comments on an earlier draft of the paper. This work was funded by SERC studentship 92307144 (A.J.T.).

\section{References}

BARTON, N. H. AND HEWITT, G. M. 1980. Hybrid zones and speciation. In: Atchley, W. R. \& Woodruff, D. (eds) Evolution and Speciation: Essays in Honor of M. J. D. White, pp. 109-145. Cambridge University Press, Cambridge.

BARTON, N. H. AND ROUHANI, S. 1991. The probability of fixation of a new karyotype in a continuous population. Evolution, 45, 499-517.
BUTLIN, R. K. 1987. Speciation by reinforcement. Trends Ecol. Evol., 2, 8-13.

CHARLESWORTH, B. AND CHARLESWORTH, D. 1973. Selection of new inversions in multilocus genetic systems. Genet. Res., 21, 167-183.

COYNE, J. A. 1992. Genetics and speciation. Nature, 355, 511-515.

DIEHL, S. R. AND BUSH, G. L. 1989. The role of habitat preference in adaptation and speciation. In: Otte, D. \& Endler, J. A. (eds) Speciation and its Consequences, pp. 345-365. Sinauer Associates, Sunderland.

FELSENSTEIN, J. 1981. Skepticism towards Santa Rosalia, or why are there so few kinds of animals. Evolution, 35, 124-138.

FRASER, A. AND BURNELL, D. 1967. Simulation of genetic systems. XII. Models of inversion polymorphism. Genetics, 37, 267-282.

GILBURN, A. S., FOSTER, S. P. AND DAY, T. H. 1992. Female mating preferences for large size in Coelopa frigida. Heredity, 69, 209-216.

GRULA, J. W. AND TAYLOR, O. R. 1980. The effects of X chromosome inheritance on mate selection behavior in the sulfur butterflies, Colias eurytheme and $C$. philodice. Evolution, 34, 688-695.

HEWITT, G. M. AND BARTON, N. H. 1980. The structure and maintenance of hybrid zones as exemplified by Podisma pedestris. In: Blackman, R. L., Hewitt, G. M. \& Ashburner, M. (eds) Insect Cytogenetics, pp. 149-170. Blackwell Scientific Publications, Oxford.

KING, M. 1993. Species evolution: the role of chromosomal change. Cambridge University Press, Cambridge.

KIRKPATRICK, M. 1982. Sexual selection and the evolution of female choice. Evolution, 36, 1-12.

LANDE, R. 1979. Effective deme sizes during long-term evolution estimated from rates of chromosomal rearrangement. Evolution, 33, 234-251.

LANDE, R. 1981. Models of speciation by selection on polygenic traits. Proc. Natl. Acad. Sci. U.S.A., 78, 3721-3725.

LEWIS, H. 1973. The origin of diploid neospecies in Clarkia. Am. Nat., 107, 161-171.

MAYNARD SMITH, J. 1991. Theories of sexual selection. Trends Ecol. Evol., 6, 146-151.

o'DONALD, P. 1962. The theory of sexual selection. Heredity, 17, 541-552.

POMIANKOWSKI, A., IWASA, Y. AND NEE, S. 1991. The evolution of costly mate preferences. I. Fisher and biased mutation. Evolution, 45, 1422-1430.

RITCHIE, M. G. 1992. Setbacks in the search for mate-preference genes. Trends Ecol. Evol., 7, 328-329.

SEARLE, J. B. 1986. Factors responsible for karyotypic polymorphism in the common shrew Sorex araneus. Proc. $R$. Soc. Lond. B, 229, 277-298.

SEGER, J. 1985. Unifying genetic models for the evolution of female choice. Evolution, 39, 1185-1193.

STURTEVANT, A. H. AND BEAdLE, G. w. 1939. An Introduction to Genetics. Saunders, Philadelphia.

WEST-EBERHARD, M. J. 1983. Sexual selection, social competition, and speciation. Q. Rev. Biol., 58, 155-183.

whiTe, M. J. D. 1978. Modes of Speciation. W. H. Freeman, San Francisco. 